\title{
The Transfer efficiency analysis and modeling technology of new non - contact power transmission equipment
}

\author{
Shi Cao ${ }^{1}$, NianSong Zhang ${ }^{1, a}$ and AiMin Wang ${ }^{2}$ \\ ${ }^{1}$ School of Mechanical Engineering, Nanjing University of Science and Technology, 210094 Nan Jing, China \\ ${ }^{2}$ School of Mechanical Engineering, Beijing Institute of Technology, 100081 Bei Jing, China
}

\begin{abstract}
Due to the shortcomings of current power transmission which is used in ultrasound assisted machining and the different transfer efficiency caused by the related parameters of the electromagnetic converter, this paper proposes an analysis model of the new non-contact power transmission device with more stable output and higher transmission efficiency. Then By utilizing Maxwell finite element analysis software, this paper studies the law of the transfer efficiency of the new non-contact transformer and compares new type with traditional type with the method of setting the boundary conditions of non-contact power supply device. At last, combining with the practical application, the relevant requirements which have a certain reference value in the application are put forward in the actual processing.
\end{abstract}

\section{Introduction}

Traditional energy transmission uses carbon brush and other physical contact approaches, these exist some shortcomings such as low speed, heating serious, wearing too fast, easy to produce EDM and so on[1-3]. In response to these problems, many scholars have proposed non-contact power supply technology. At present, non-contact power transmission devices are divided into two categories: sliding type and rotary type[4]. In the actual processing applications, rotating type occupies a dominant position. The non-contact power transmission devices of traditional rotary type up and down are $360^{\circ}$ core, for the new rotary non-contact power transmission device, its first half is the $120^{\circ}$ core, the second half is the $360^{\circ}$ core. Some scholars have improved non-contact power transmission efficiency through the finite element method, however, these studies are only based on the traditional non-contact power transmission device, the new non-contact power transmission device is rarely involved[5-6].

This paper presents an analysis model of the new non-contact power transmission device, through researching the influence of three parameters which are core gap, core offset and core thickness having effect on non-contact power transmission efficiency, the curve whose relationship is between the relevant parameters and transfer efficiency is drawing in the text so that it can give the specific transfer efficiency. The three machining parameters are validated by finite element analysis, which provides the theoretical basis for the subsequent parameter optimization.

${ }^{\mathrm{a} C}$ Corresponding author :zns@ustc.edu.cn 


\section{Maxwell simulation modeling}

Maxwell is a powerful electromagnetic simulation tool, which is mainly used to analyze two-dimensional and three-dimensional electromagnetic components, such as motors, transformers, actuators and other electrical and mechanical and electrical equipment, where applies in the automotive, military, aerospace and industrial.

Magnetic materials plays an important part in non-contact power transmission devices, today's magnetic components have a very wide operating range where can operate over the audio range up to the megahertz range. The magnetic permeability of a magnetic material is the standard to measure how easy it is to be magnetized. The value can be expressed as:

$$
\mu=\frac{B}{H}
$$

Among them, $B$ represents the magnetic flux density and $H$ is the magnetic field strength. Nowadays, the general choice is the ferrite core material. The ferrite is a homogeneous ceramic material consisting of iron oxide and other oxides. A soft ferrite can be divided into two main categories: manganese-zinc and nickel-zinc. In each category, changing the chemical composition or manufacturing method can create more different categories. Among them, the power ferrite is a kind of the manganese-zinc ferrite, which mainly uses in high-power transmission, where operates from audio to hundreds of megahertz. So that in this paper, the power ferrite is selected in the core material. For the sake of calculating simply, a method that the window area of the core is divided by an equal current density according to the power handling capability of the coil is used in this paper. The processing power of the primary coil is $P_{i n}$, and the power of the secondary coil to the load is $P_{0}$.

The total apparent power is calculated as:

$$
P_{t}=P_{t}+P
$$

In the above formula, $P_{i n}=\frac{P_{0}}{\eta}, P_{t p}=P_{i n} P_{a}$, In addition, through calculation formula of the electric state and the core geometry constant:

$$
\begin{gathered}
K_{\varepsilon}=0.145 K_{f}^{2} f^{2} B_{A C}^{2} \times 10^{-4} \\
K_{g}=\frac{P_{t}}{2 K_{\varepsilon} \alpha}
\end{gathered}
$$

Selecting the core which has a similar geometric constants $K_{g}$ to obtain the number of winding turns $N_{p}$, current density $J$, input current $I_{i n}$ and coil resistance $R_{p}$ respectively:

$$
\begin{gathered}
N_{p}=\frac{V_{p} \times 10^{4}}{A_{C} B_{A C} f K_{f}} \\
J=\frac{P_{t} \times 10^{4}}{K_{f} K_{u} B_{A C} f A_{p}} \\
I_{i n}=\frac{P_{0}}{V_{i n} \eta} \\
R_{p}=\operatorname{MLT}\left(N_{p}\right) \frac{\mu \Omega}{c m} \times 10^{-6}
\end{gathered}
$$

The finite element model of the new non-contact power transmission device is built on the Maxwell software platform. The transfer efficiency of the non-contact power transmission device is mainly analyzed from the variation of the core gap, the thickness of the magnetic core and the thickness of the magnetic core. The transient field solver module was first selected and then the 3D model of the non-contact power transmission device was built according to Figures 1 and the dimensions listed in Table 1. Afterwards, setting the boundary conditions, $20 \mathrm{KHz}$ ultrasonic 
frequency AC signal is applied to the primary coil. The number of coil turns are 100.Establishing a three-dimensional model shown in Figure 2.

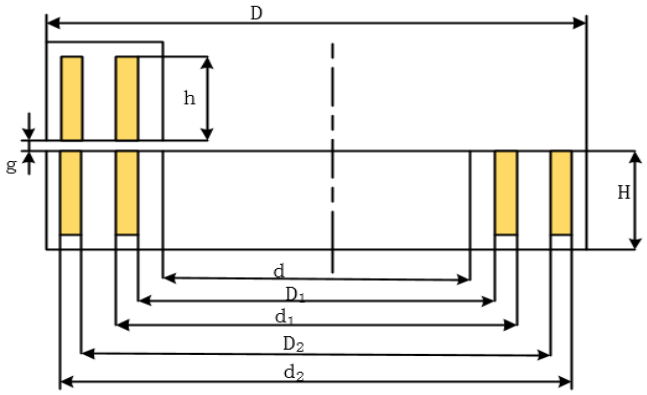

Figure 1. Sectional view of new non-contact power transmission device

Table 1. Dimensions of non - contact power transmission devices

\begin{tabular}{|c|c|c|}
\hline $\begin{array}{c}\text { Parameter } \\
\text { name }\end{array}$ & symbol & $\begin{array}{c}\text { Parameter } \\
\text { size (mm) }\end{array}$ \\
\hline $\begin{array}{c}\text { Core outer } \\
\text { diameter }\end{array}$ & $\mathrm{D}$ & 100 \\
\hline $\begin{array}{c}\text { Core inside } \\
\text { diameter }\end{array}$ & $\mathrm{d}$ & 65 \\
\hline Core thickness & $\mathrm{H}$ & 20 \\
\hline $\begin{array}{c}\text { First winding } \\
\text { outer diameter }\end{array}$ & $D_{1}$ & 70 \\
\hline $\begin{array}{c}\text { First winding } \\
\text { inside } \\
\text { diameter }\end{array}$ & $d_{1}$ & 78 \\
\hline $\begin{array}{c}\text { Second } \\
\text { winding outer } \\
\text { diameter }\end{array}$ & $D_{2}$ & 88 \\
\hline $\begin{array}{c}\text { Second } \\
\text { winding inside } \\
\text { diameter }\end{array}$ & $d_{2}$ & 96 \\
\hline $\begin{array}{c}\text { Winding } \\
\text { thickness }\end{array}$ & $\mathrm{h}$ & 15 \\
\hline Core clearance & $\mathrm{g}$ & 1 \\
\hline
\end{tabular}

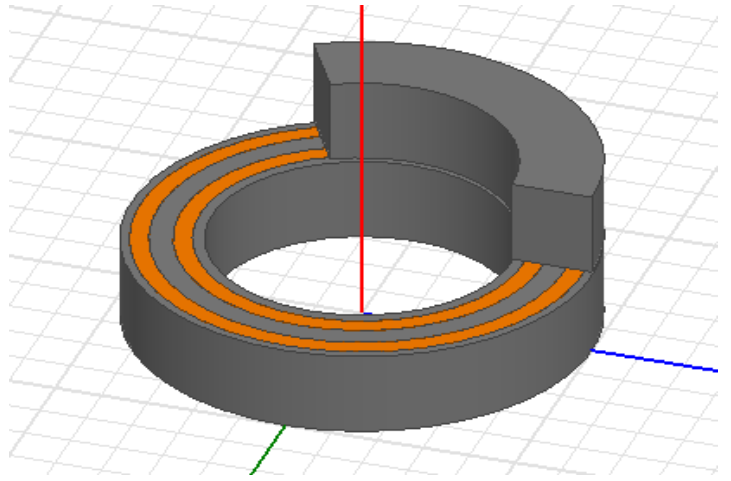

Figure 2. Three - dimensional model of non - contact power transmission device

When the secondary winding speed gradually increases, the current amplitude of the primary coil and secondary coil remain unchanged, the rotational speed of the secondary coil has little effect on the efficiency of the non-contact power transmission device. Because of the characteristics of the ferrite 
core, it can react instantaneously to the changes in the tens or even hundreds of hertz, moreover at the speed of $2000 \mathrm{rev} / \mathrm{min}$. Therefore, in this paper, you can ignore the impact of the speed on transmission efficiency.

As can be seen in Figure 3, the input current of the primary coil and the induced current of the secondary coil have the same frequency and opposite phase angle. When the input current amplitude of the primary coil reaches $0.8 \mathrm{~A}$, the first secondary coil output current is $0.34 \mathrm{~A}$ and the second secondary coil output current is $0.28 \mathrm{~A}$. This is due to the presence of magnetic flux leakage, resulting in that the induction current of secondary coil is smaller than the input current of primary coil.

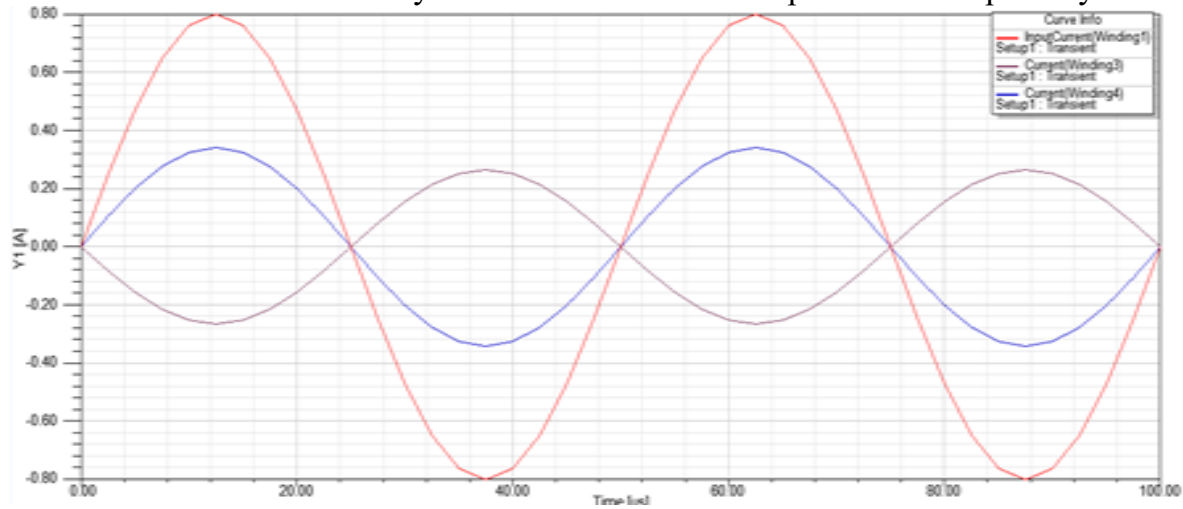

Figure 3. Primary and Secondary Coil Current

\section{Effect of magnetic core spacing}

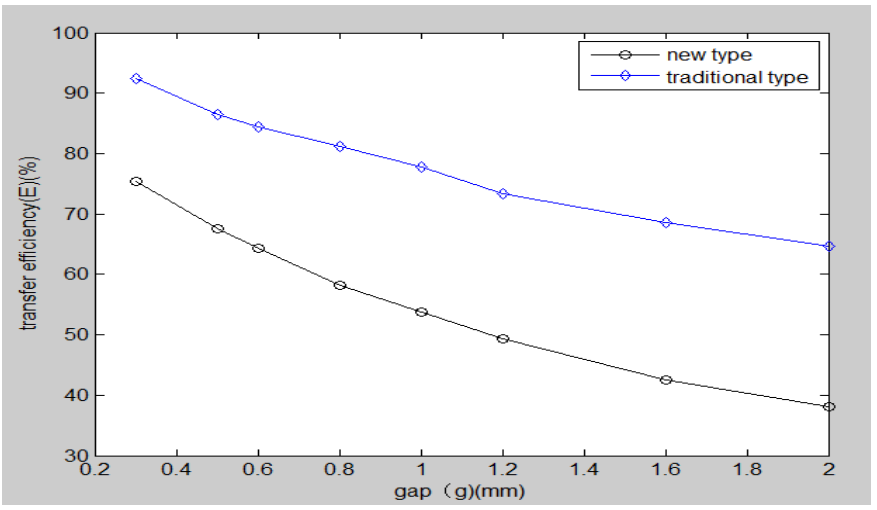

Figure 4. Transmission Efficiency with the change of Core Spacing

As shown in Figure 4, when the other parameters remain unchanged, When the two core spacing of the non-contact power transmission device are changed in pitch from $0.3 \mathrm{~mm}$ to $2 \mathrm{~mm}$, whether it is a new non-contact power transmission device or a traditional non-contact power transmission device, the transmission efficiency reduces along with the growth of the core spacing. This is because with the increasing of magnetic core spacing, reluctance and magnetic leakage increases, mutual inductance decreases, resulting in the decreasing of the coupling coefficient between original and secondary winding, making the transfer efficiency decrease. In the same core spacing, the transfer efficiency of new non-contact power transmission device is smaller than that of the traditional type, moreover, when the two non-contact power transmission device core spacing are in the $0 \mathrm{~mm}$ to $1 \mathrm{~mm}$, the transmission efficiency changes mostly severe, the change of the transmission efficiency begins to slow down as it continues to increase. When the core spacing increases to a certain extent, the mutual inductance may be very weak, which can't produce the induced current, the transmission efficiency of the non-contact power transmission device will be 0 . 
Therefore, the transmission effect is the best when the distance is 0 between the original and the secondary winding gap theoretically. However, in practice, due to processing and installed problems, the original, the secondary winding spacing of the non-contact power transmission device can't be infinitely small, the selected core spacing is generally within $0.6 \mathrm{~mm}$.

\section{Effect of core offset}

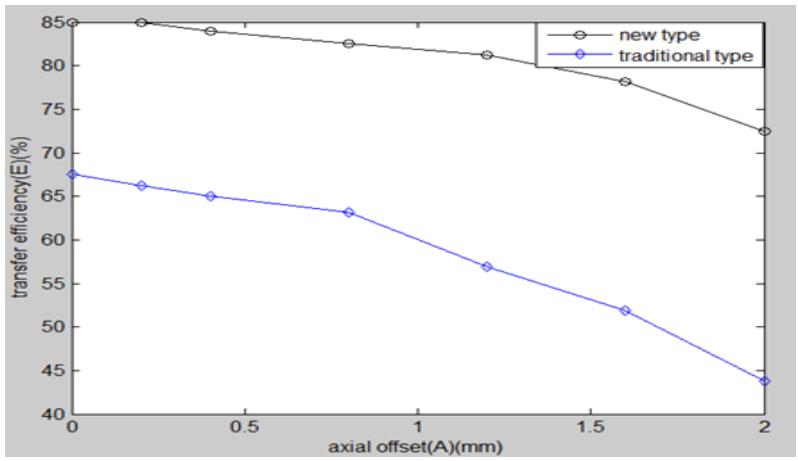

Figure 5. Transmission Efficiency with the change of core offset

In the actual processing, as in the processing and assembly of the handle, core, the core coat and some other parts, there is likely to happen coaxial error, leading that the primary winding and secondary winding are not aligned. Whether the non-contact power transmission system working conditions will change in case of this axis offset, and the impact is how much, these are all obtained by Maxwell simulation.

In the above figure, the transfer efficiency of the two non-contact power transmission devices is reduced when the two core offset of the non-contact power transmission device changes from 0mm to $2 \mathrm{~mm}$ while the other parameters are kept unchanged. This is because with the increasing of core offset, reluctance and magnetic leakage increases, mutual inductance decreases, resulting in the decreasing of the coupling coefficient between original and secondary winding, making the transfer efficiency decrease. In addition, it can be seen that the traditional non-contact power transmission device has little change in the transfer efficiency when the offset is small. As the offset continues to increase, the transfer efficiency decreases slowly, and later becomes fast, which is similar to the new non-contact power transmission device.

In the actual processing of parts, it needs to ensure the core offset that the error of concentricity isn't more than $0.3 \mathrm{~mm}$ so that a higher energy transfer can be achieved.

\section{Effect of core thickness}

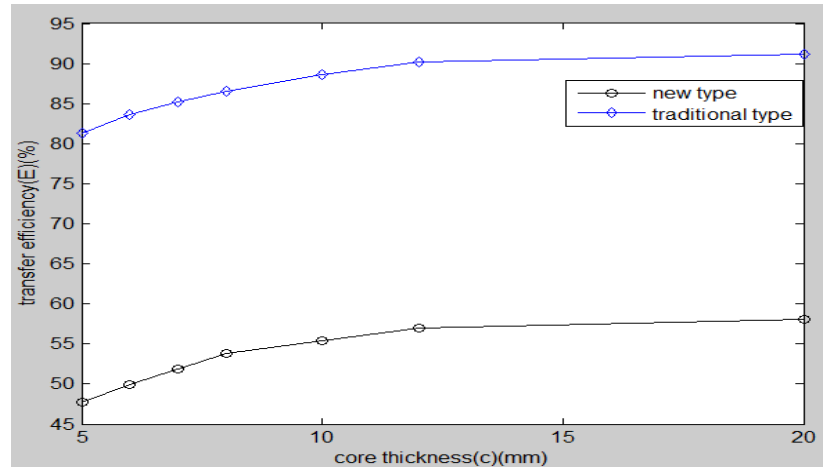

Figure 6. Transmission Efficiency with the change of core thickness 
It can be seen from Figure. 6 that the increase of the core thickness can effectively improve the transfer efficiency when the other parameters are unchanged whether traditional or new non-contact power transmission devices. This is because the thicker core has a good poly-magnetic effect, thereby reducing the leakage of magnetic energy into the air. According to the fitting curve in the figure, when the thickness is less than $10 \mathrm{~mm}$, the increase rate of transmission efficiency is relatively large. As the thickness continues to increase, the increase rate of transmission efficiency begins to slow down gradually, which indicates that the variation of transmission efficiency and core thickness is convergent.

In the actual processing of parts, the core is generally maintained at a thickness of $10 \mathrm{~mm}$ or more appropriate.

\section{Conclusion}

In this paper, the non-contact power transmission device is used as a model, utilizing Maxwell software to obtain relationship curve between the three parameters and transmission efficiency belong to the core. By comparing the new and traditional non-contact power transmission devices, It analyzes the factors affecting the transfer efficiency in the actual processing in order to get the maximum energy transferred in the ultrasonic vibration processing.

In the same conditions, although the transfer efficiency of the traditional non-contact power transmission device is higher than that of the new non-contact power transmission device, however, on the one hand, the transfer efficiency of new type has been basically meet the production requirements in the industrial application, on the other hand, it isn't convenient to change cutleries in traditional type, which will reduce the production efficiency greatly.

So the non-contact power transmission device is currently in the direction of changeable cutlery. The reasonable matching network with experiments will be implemented in future work.

\section{References}

1. Wen Yuan, Zhili Long, Jianguo Zhang, et al. The application of new non-contact power transducer in ultrasonic machining, Transactions of China Electrotechnical Society[J], 30(10):53-57(2015)

2. Valtchev S, Borges B, Brandisky K, et al. Resonant contactless energy transfer with improved efficiency[J]. IEEE Transactions on Power Electronics, 24(3):685-699(2009)

3. Yajun Li, Xinquan Lai, Qiang Ye, et al. High efficiency and low electromagnetic interference boost DC-DC converter. Journal of Semiconductors[J], 35(4):1-8(2014)

4. Qiang Ye, Jie Liu, Bing Yuan, et al. On-ship frequency compensation with a dual signal path operational transconductance amplifier for a voltage mode control DC/DC converters. Journal of Semiconductors, 33(4):045006(2012)

5. Reiter T, Polenov D, Probstle H, et al. PWM dead time optimization method for automotive multiphase DC/DC-converters. IEEE Trans Power Electron, 25(6):1604(2010)

6. M Du, H Lee, J Lin. A 5-MHz 91\% peak-power-efficiency buck regulator with auto-selectable peak and valley-current control IEEE J Solid-State Circuit, 46(8):1928(2011) 